\title{
Structural Studies of the Purine and SAM Binding Riboswitches
}

\author{
S.D. Gilbert, ${ }^{*}$ R.K. Montange, ${ }^{*}$ C.D. Stoddard, and R.T. Batey \\ Department of Chemistry and Biochemistry, University of Colorado, Boulder, Colorado 80309-0215
}

\begin{abstract}
Riboswitches are recently discovered genetic regulatory elements found in the $5^{\prime}$-untranslated regions of bacterial mRNAs that act through their ability to specifically bind small-molecule metabolites. Binding of the ligand to the aptamer domain of the riboswitch is communicated to a second domain, the expression platform, which directs transcription or translation of the mRNA. To understand this process on a molecular level, structures of three of these riboswitches bound to their cognate ligands have been solved by X-ray crystallography: the purine, thiamine pyrophosphate (TPP), and $S$-adenosylmethionine (SAM-I) binding aptamer domains. These studies have uncovered three common themes between the otherwise different molecules. First, the natural RNA aptamers recognize directly or indirectly almost every feature of their ligand to achieve extraordinary specificity. Second, all of these RNAs use a complex tertiary architecture to establish the binding pocket. Finally, in each case, ligand binding serves to stabilize a helix that communicates the binding event to the expression platform. Here, we discuss these properties of riboswitches in the context of the purine and SAM-I riboswitches.
\end{abstract}

In mRNA, noncoding regions contain an enormous amount of information that is crucial for proper gene expression. It has long been recognized that bacterial gene regulation is controlled at the translational and transcriptional levels via proteins that bind structures and sequences in mRNA 5'UTRs (untranslated regions). The classic example of this form of regulation is that of the TRAP protein, which, in the presence of high levels of tryptophan, binds the $5^{\prime} \mathrm{UTR}$, affecting the formation of regulatory stem-loop structures (Babitzke 2004). At the translational level, a number of ribosomal proteins associate with elements in the $5^{\prime}$ UTR of their own mRNAs that are structurally similar to their rRNA-binding sites, occluding important elements for formation of the translational initiation complex (Zengel and Lindahl 1994). However, there are a number of $5^{\prime}$ UTRs that display strong conservation in their sequence and secondary structure that could not be associated with any known protein or other regulatory factor, and thus their function and mechanism of action remained elusive.

The first of these "orphan" elements to be extensively characterized was one found by Henkin and Grundy in the $5^{\prime}$ UTR of operons coding for genes involved in sulfur metabolism pathways (Grundy and Henkin 1998). The striking feature of this element, dubbed the S-box, was its apparent ability to fold into two mutually exclusive structures, ostensibly in the presence of a regulatory factor. It was thus postulated that the S-box uses these structures to regulate transcription through the ability of some unidentified factor to bind the $5^{\prime}$ UTR in the presence of methionine. Almost concurrently, another RNA element was discovered, called the RFN element, that was found upstream of genes encoding proteins responsible for riboflavin synthesis in gram-positive bacteria (Gelfand et al. 1999). The RFN element, in addition to forming

*These two authors contributed equally to this work. mutually exclusive stem-loop structures, contains five strongly conserved helical regions and was found not only in the $5^{\prime}$ UTR of one gene or operon across different genomes, but also within different genes and operons within one genome. Similar to the S-box, no protein factor could be identified that bound the RFN element, and so it was hypothesized that the mRNA may directly bind the reaction product of these genes, a small-molecule metabolite. Gelfand and coworkers speculated that these conserved sequences fold into structures similar in function to RNA aptamers that bind small molecules through in vitro selection methods (SELEX). Studies of a third element, the thi box, found in the $5^{\prime}$ UTRs of thiamine biosynthetic genes, yielded further information that a small molecule (in this case, thiamine pyrophosphate) can bind RNA in the absence of a protein cofactor, thereby modulating the formation of downstream secondary structure and gene expression (Miranda-Rios et al. 2001).

In 2002, the Breaker and Nudler laboratories independently and concurrently conducted definitive studies proving this hypothesis. The first of a series of publications describing what has become known as riboswitches was an investigation of the adenosylcobalamin (vitamin $\mathrm{B}_{12}$ ) associated element (Nahvi et al. 2002), closely followed by confirmation of the RFN (Mironov et al. 2002; Vitreschak et al. 2002), thi box (Winkler et al. 2002b), and S-box elements (McDaniel et al. 2003; Winkler et al. 2003). These mRNAs were shown to directly bind smallmolecule metabolites and alter their conformation in response to the binding event using a number of biochemical techniques, most notably the "in-line" probing method pioneered by the Breaker laboratory (McDaniel et al. 2003; Winkler et al. 2003). Furthermore, it was demonstrated that mutations disrupting Watson-Crick paired helices in the conserved regions abrogated ligand binding, which could be restored by compensatory mutations that reestablished the helix. This behavior is a 


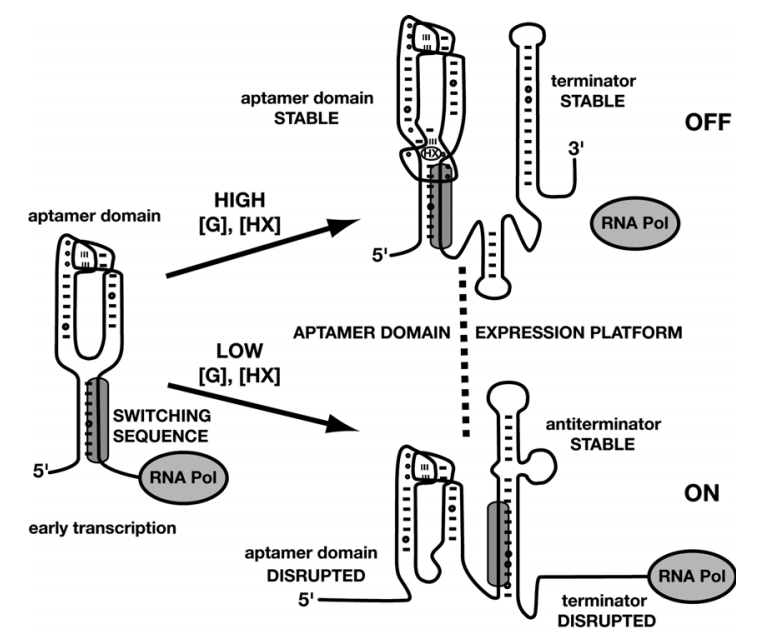

Figure 1. Schematic of transcriptional repression by the purine riboswitch. Early in transcription, the first element of a riboswitch that is formed is the aptamer domain (left) that binds a specific small-molecule metabolite. If a sufficient metabolite concentration is present in the cell, it binds to the aptamer and stabilizes the incorporation of a "switching sequence" into this domain (right top), fating the mRNA to form a rho-independent terminator, causing transcription to halt. In the absence of metabolite, the switching sequence is used by the expression platform to form an antiterminator element (right bottom), allowing transcription to proceed and the gene to be expressed.

hallmark of RNAs whose activity is dependent on higherorder folded structures. Finally, it was clearly shown that the binding of the ligand to the "aptamer domain" of the riboswitch is communicated to an "expression platform" (Fig. 1) comprising a secondary structural switch that is used to direct gene expression.

Bioinformatic approaches, coupled with biochemistry, have resulted in the identification of a number of other riboswitches in rapid succession (for review, see Winkler and Breaker 2005). This has been primarily achieved using the criteria that riboswitches are found in the 5'UTR of genes, that they contain conserved sequences and secondary structures that are conserved across a segment of bacterial phylogeny, and that the effector molecule is closely related to the function of the gene product in which they are found. To date, riboswitches that use thiamine pyrophosphate (Rodionov et al. 2002; Winkler et al. 2002b; Sudarsan et al. 2003a), $S$-adenosylmethionine (Grundy and Henkin 1998; Mandal et al. 2003; Barrick et al. 2004), adenine and guanine (Mandal et al. 2003), glycine (Mandal et al. 2004), lysine (Rodionov et al. 2003; Sudarsan et al. 2003b), glucosamine-6-phosphate (Winkler et al. 2004), flavin mononucleotide (Vitreschak et al. 2002; Winkler et al. 2002a), coenzyme $B_{12}$ (Nahvi et al. 2002; Mandal et al. 2003), and $\mathrm{Mg}^{2+}$ (Cromie et al. 2006) have been identified. Currently, there are also a number of conserved secondary structures in bacterial mRNA for which there are still no identified effector molecules (Barrick et al. 2004; Corbino et al. 2005). Therefore, it is very likely that this list will continue to expand.

In this paper, we describe our efforts to determine the structure of several of these metabolite-binding RNA elements by X-ray crystallography. Through a structural investigation of the aptamer domains of the purine and $S$-adenosylmethionine (S-box) riboswitches, we sought to address two fundamental questions. First, how do these mRNAs specifically bind their metabolite? Associated with this question is how these mRNAs achieve such high discrimination between their cognate ligand and a number of chemically similar metabolites also found in the cell. Second, how does the aptamer domain communicate the ligand-binding event to the expression platform? This is the crucial feature of the riboswitch that allows it to act as an effective regulator of gene expression.

\section{PURINE RIBOSWITCH}

The purine riboswitch (also known as the G-box and Abox) is a regulatory element found upstream of a number of genes involved in purine biosynthesis and transport. It comprises a series of conserved sequences spread over an approximately 60-nucleotide region (Mandal et al. 2003; Mandal and Breaker 2004b). Biochemical characterization revealed that it folds into a three-helical structure with the conserved nucleotides localizing to two terminal loops and a three-way junction (Fig. 2A). It was also noticed that a single pyrimidine residue covaried with the ligand; in guanineresponsive elements, it is always a cytosine, and in adenine-responsive elements, it is always uracil (Fig. 2a, asterisk). This observation suggested that purine specificity is dictated in large part by the ability of the nucleobase to form a Watson-Crick base pair with this pyrimidine.

\section{Structure Determination}

To determine the structure of this RNA element bound to its cognate ligand by X-ray crystallography, we used the Gbox from the xpt-pbuX operon of Bacillus subtilis (Mandal et al. 2003). One of the most powerful strategies in RNA

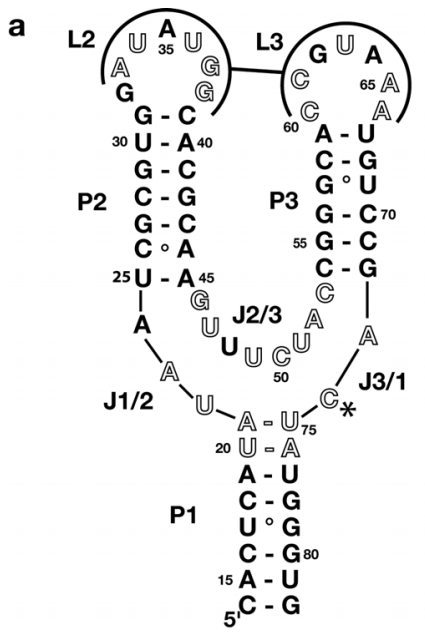

b

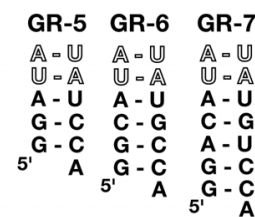

GR-8 GR-9 GR-11

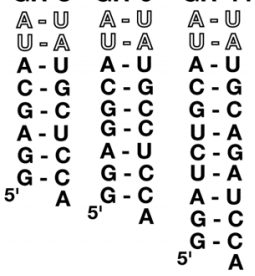

Figure 2. (a) Secondary structure of the purine riboswitch aptamer domain of the $x p t-p b u X$ operon from B. subtilis. Nucleotides that are outlined are $>90 \%$ conserved, and the starred nucleotide represents the pyrimidine that is cytosine in the guanine riboswitches and uridine in adenine riboswitches. (b) Sequence variants of the P1 helix introduced in a limited library of variants that were constructed and tested for crystallizability; construct "GR7" readily produced diffraction-quality crystals. 
crystallography with respect to finding a sequence that readily crystallizes and yields high-quality diffraction data is to systematically vary peripheral elements, such as helices and loops (Ke and Doudna 2004). Unfortunately, because highly phylogenetically conserved nucleotides are interspersed throughout the RNA, the only region that could be altered without affecting the ability to bind ligand is the length of the P1 helix (Fig. 2b) (Mandal et al. 2003). Thus, in our initial survey, we used a series of constructs in which the length of this helix was between 6 and $11 \mathrm{bp}$. To circumvent any potential issues with refolding the RNA, we prepared all of the RNA using a newly developed native purification system that allowed us to rapidly purify approximately 10-mg quantities in 2-3 hours (Kieft and Batey 2004). Once prepared and bound to hypoxanthine, a soluble derivative of guanine that also binds the G-box, the RNAs were subjected to a suite of standard commercially available sparse matrices (PEG-Ion, Natrix, Crystal Screen, and Nucleic Acid Mini Screen; Hampton Research). One of the constructs (GR7, Fig. 2b) rapidly yielded crystals under several conditions in the Nucleic Acid Mini Screen (conditions 1-4). In particular, it was found that the G-box required $10-20 \mathrm{~mm}$ cobalt hexaammine to yield crystals that diffracted to approximately $3 \AA$.

Along the route toward obtaining data that would yield phase information and allow calculation of an interpretable electron density map, we encountered several issues. First was the ability to generate a crystal form that allowed us to collect quality data sets. Our initial crystal form (Fig. 3a) was of the P2 space group, with a very large unit cell that resulted in a diffraction pattern with very closely spaced reflections. Resolving these reflections for precise quantification of their volumes was almost impossible without the aid of a synchrotron X-ray source. Consequently, we sought other forms that would be more amenable to analysis with our home source. A second form was achieved by switching the growth temperature to $4^{\circ} \mathrm{C}$, yielding crystals of the P3 22 space group (Fig. 3b). Whereas these crystals had a smaller unit cell, analysis of the data clearly indicated that they were hemihedrally twinned, rendering them useless for obtaining phase information. Using microseeding approaches (Stura and Wilson 1990), we were able to obtain a third form, which was not twinned, but contained a C2 space group and a small unit cell. This was the form used in all subsequent work.

Obtaining phase information via the incorporation of heavy atoms is another serious issue in RNA X-ray crystallography structure determination. In protein crystallography, it is routine to use selenomethionine to incorporate a heavy atom that is suitable for obtaining experimental phases with the multiwavelength anomalous dispersion (MAD) method (Hendrickson and Ogata 1997). Although this advance has enormously facilitated protein structure determination, the same advance has not been made for RNA structure determination. Incorporation of bromine by in vitro transcription with 5-bromouridine triphosphate (Kieft et al. 2002; Zhang and Doudna 2002) or synthetically using 2'-methylselenosubstituted nucleosides has been successful (Teplova et al. 2002; Carrasco et al. 2004), but is difficult to achieve with larger RNAs (>50 nucleotides) (Hobartner et al. 2005). Another approach is to use selenomethionine-labeled U1A
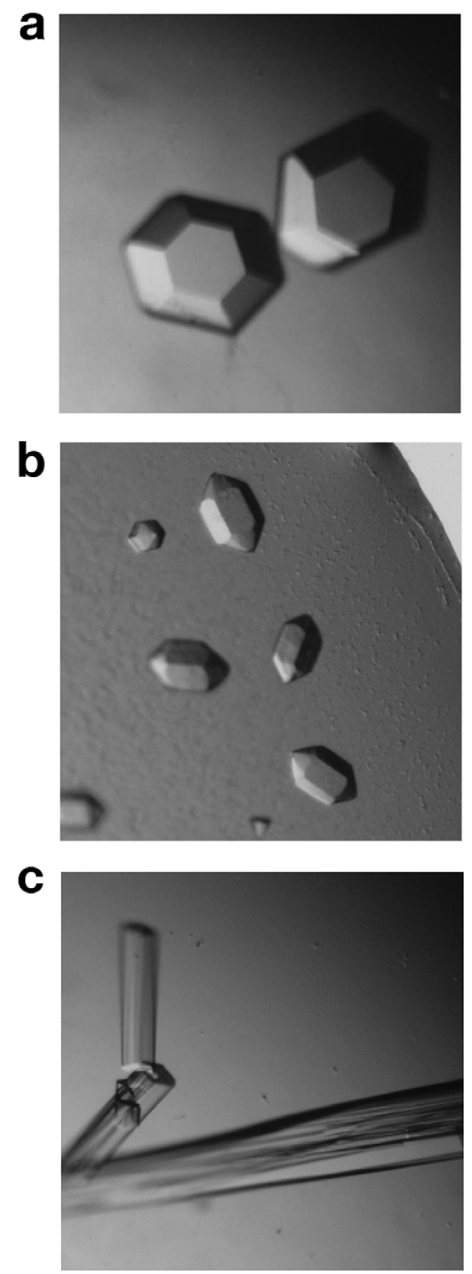

Figure 3. Crystals of the GR7 construct. The first crystal form $(a)$ had one large unit cell dimension (P2 space group; $\mathrm{a}=71.5 \AA$, $\mathrm{b}=275.8 \AA, \mathrm{c}=71.9 \AA, \alpha=\gamma=90^{\circ}, \beta=119.6^{\circ} ; \sim 3.0 \AA$ diffraction limit) that made crystals difficult to work with due to closely spaced reflections. The second form obtained $\left(\mathrm{P} 3_{1} 22\right.$ space group; $\mathrm{a}=\mathrm{b}=117.3 \AA, \mathrm{c}=90.0 \AA, \alpha=\gamma=90^{\circ}, \beta=120^{\circ} ; \sim 2.5 \AA$ diffraction limit) were shown to be twinned using the algorithm of Yeats and coworkers. The third form (C2 space group; $\mathrm{a}=132.3 \AA$, $\mathrm{b}=35.3 \AA, \mathrm{c}=42.2 \AA, \alpha=\gamma=90^{\circ}, \beta=91^{\circ} ; \sim 1.9 \AA$ diffraction limit) is what was used to solve the crystal structure.

bound to the RNA as a carrier of heavy atoms for phasing (Ferre-D'Amare and Doudna 2000), an approach that has been successfully applied to a number of ribozyme structures (Ferre-D'Amare et al. 1998; Rupert and FerreD'Amare 2001; Adams et al. 2004). However, given the predicted fold of the G-box, it was not clear how to engineer a U1A-binding site into the RNA without perturbing the ligand-binding site. After a number of heavy atom soaking trials with standard compounds such as platinum, gold, and mercury derivatives, we were unable to generate native and derivative data sets that were sufficiently isomorphous to be able to solve the phase problem using the multiple isomorphous replacement (MIR) method.

To overcome this difficulty, we turned to a recently established technique called single-wavelength anomalous dispersion (SAD) phasing (Rice et al. 2000; Dauter et al. 2002). In this method, data is only collected at a single 
wavelength of $\mathrm{X}$-ray radiation, and the phase ambiguity is broken by visual inspection of the electron density maps generated from the two solutions. As a heavy atom, we decided to rely on cobalt hexaammine, which was a required component in the mother liquor and was likely bound to the RNA, as the crystals were a faint orange in color. The absorption peak of cobalt is very close to the wavelength of $\mathrm{X}$-ray radiation from a rotating copper anode source (1.608 $\AA$ vs. $1.542 \AA$ ); at this wavelength, cobalt is a weak anomalous scatterer with 3.61 electrons contributing to the scattering. We performed an inverse-beam experiment on crystals that were grown and cryoprotected in $12 \mathrm{~mm}$ cobalt hexaammine (the crystals were not backsoaked to remove cobalt from the mother liquor), collecting $20^{\circ}$ wedges with $0.5^{\circ}$ oscillation per frame. Because the crystals showed significant mosaicity $\left(\sim 0.8^{\circ}\right)$, we had to use D*TREK (Pflugrath 1999) to accurately merge partial reflections and integrate the volume of the entire reflection. Using SOLVE (Terwilliger 2003), we initially sought a solution containing 4 heavy atoms, which was rapidly found by the program. Further sites were found by incrementally searching for more sites, until a maximum of 10 strong sites were found. This solution was imported into crystallography and NMR system (CNS) (Brunger et al. 1998) for calculation of experimental phases, density modification using a solvent flipping routine, and calculation of an electron density map for each of the two possible solutions (the heavy-atom solution and its mirror image). Only one solution yielded a weakly interpretable map in which density for the backbone and a number of bases could be observed.

To build the model in this map of moderate quality, we chose to initially build uridine into bases that had the approximate shape of a pyrimidine, and adenine into regions that looked like purines. Using this strategy, we were able to build approximately $60 \%$ of the model. At this point, we performed one round of energy minimization and B-factor refinement on the model and used the resulting model to calculate a new phase-combined map that displayed clear electron density for all residues in the G-box and for which the sequence register could be unambiguously obtained.

\section{Structure of the Purine Riboswitch}

The architecture of the purine riboswitch is defined by two regions of extensive noncanonical base-pairing (Fig. 4) (Batey et al. 2004; Serganov et al. 2004). A distinctive side-by-side arrangement of the P2 and P3 helices is created by an unusual loop-loop (L2-L3) interaction that is formed by two sets of base quartets involving eight universally conserved nucleotides. Each quartet comprises a Watson-Crick pair between nucleotides from each loop and a noncanonical base pair docked in the minor groove of the Watson-Crick pair. These two quartets adopt an identical configuration in both the B. subtilis xpt-pbuX guanine riboswitch and the Vibrio vulnificus add adenine riboswitch. A set of two further noncanonical base pairs are found in the guanine riboswitch at the top of the L2-L3 interaction that are absent in the adenine riboswitch; nucleotides involved in these pairs are significantly less phylogenetically conserved, suggesting that they are not

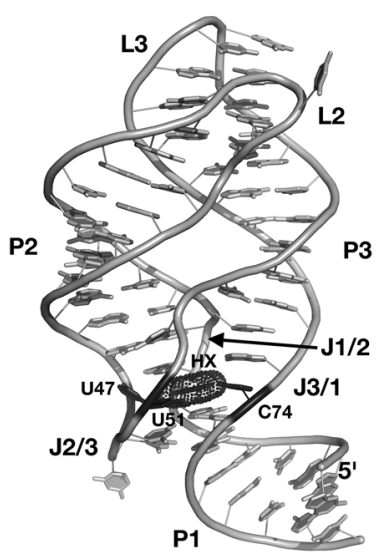

Figure 4. Global fold of the guanine riboswitch aptamer domain (PDB 1U8D). The binding pocket for the guanine analog hypoxanthine (HX, emphasized with a surface representation) is embedded within the three-way junction formed by $\mathrm{J} 1 / 2, \mathrm{~J} 2 / 3$, and $\mathrm{J} 3 / 1$. The ligand is bound through hydrogen-bonding interactions with U51 and C74 (dark gray).

required for stabilization of the tertiary contact. Although this element of structure is not directly involved in ligand recognition, elimination of this feature from the RNA by conversion of each loop into a stable UUCG tetraloop completely abolishes ligand binding (Batey et al. 2004).

Ligand binding is accomplished using a complex architecture formed by the central three-way junction. This region comprises two base triples that flank each side of the central ligand-binding pocket. Ligand recognition is achieved via a base quadruple in which the purine forms a Watson-Crick pair with a pyrimidine (C74), N3 and N9 of the nucleobase are recognized by U51, and N7 by the 2'-hydroxyl group of U22 (Fig. 5a).

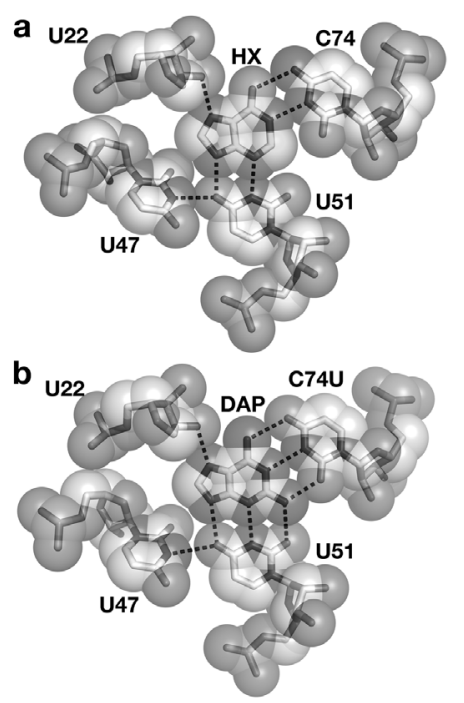

Figure 5. Hydrogen-bonding interactions involved in guanine and adenine recognition. (a) Hypoxanthine (HX) is bound using a series of hydrogen bonds to a pyrimidine-rich binding pocket including the ribose sugar of $\mathrm{U} 22$ and the bases of U47, U51, and C74 (PDB 1U8D). (b) Binding of the adenine analog 2,6diaminopurine (DAP) to the guanine riboswitch containing a single point mutation $\mathrm{C} 74 \mathrm{U}$, demonstrating that purine specificity is completely dictated by pyrimidine 74 (PDB 2B57). 
U51 is buttressed through hydrogen bonding to U47 to complete the quartet. This structure also clearly demonstrates how the purine riboswitch could accomplish a specificity swap from guanine to adenine through the change of a single nucleotide (Y74); all other contacts to the nucleobase are identical for the two ligands. Crystallization of the $x p t-p b u X$ guanine riboswitch with a single C74U mutation with the adenine analog 2,6diaminopurine directly demonstrated that the switch involves no other changes in the riboswitch architecture (Fig. 5b) (Gilbert et al. 2006).

\section{Mechanism of Ligand Binding}

This structure begs a question about the ligand-RNA interaction: How does the ligand manage to bind to a site that becomes buried within the center of the threeway junction? The essence of this problem lies in the fact that the nucleobase is approximately $98 \%$ solventinaccessible with no channel that would allow it entry from bulk solvent. Intrinsic to this question is, What exactly is the free state of the RNA? NMR spectroscopy and in-line probing clearly indicate that the three-way junction is conformationally flexible, lacking a single discrete structure (Mandal et al. 2003; Noeske et al. 2005). Yet, this element cannot be completely disorganized, as the ligand needs to recognize and dock with some element of the junction.

As previously noted, residues of each strand in the junction contact the ligand. Footprinting of the RNA by in-line probing yields information about the dynamic environment of each residue in the unbound state, indicating that the $\mathrm{J} 3 / 1$ strand is relatively rigid when compared with $\mathrm{J} 1 / 2$ and $\mathrm{J} 2 / 3$. The $\mathrm{J} 3 / 1$ strand presents a pyrimidine residue that we call the "specificity pyrimidine" (C74 in the xpt-pbuX G-box), which forms a Watson-Crick pair with the ligand. Using thermodynamic data collected from an extensive number of purine nucleobase analogs, it is clear that the hydrogen bonds between the pyrimidine and the ligand are extremely important for productive binding (Gilbert et al. 2006). A minimum of two hydrogen bonds is required in this interaction to achieve riboswitch recognition of its ligand. Furthermore, the structure indicates that the ligand does not stack upon neighboring bases in the ligand-binding pocket, underscoring the importance of hydrogen bonding in binding. Supporting this idea, when U48, a residue that is flipped into the solvent in the bound structure, is substituted with the fluorescent base 2-aminopurine, a large change in fluorescence (nearly $100 \%$, correlated to the concentration of riboswitch in solution) is observed as the ligand is bound up by the riboswitch, consistent with a significant conformational change (Gilbert et al. 2006). From this evidence, we propose a binding mechanism in which the purine ligand is first recognized by the specificity pyrimidine $\mathrm{C} 74$, prompting the subsequent organization of the three-way junction.

To effectively regulate gene expression, ligand binding to the aptamer domain must be communicated to the expression platform. A consequence of the mechanism of ligand recognition that we have proposed is that ligand binding and stabilization of the P1 "communication helix" is directly and intimately coupled (Gilbert et al. 2006). Only upon recognition of the correct ligand by pyrimidine 74 will J2/3 clamp down to complete the (Purine-Y74)•U51•U47 quadruple. Concurrently, the two base triples between Watson-Crick pairs in the P1 helix and U49 and C 50 of J2/3 are formed, which is instrumental in stabilizing the $3^{\prime}$ strand of the P1 helix ("switching sequence," Fig. 1) against incorporation into the antiterminator in the expression platform. In this way, ligand binding is highly coupled to the structural switch on the expression platform, the central feature of riboswitch function.

\section{S-ADENOSYLMETHIONINE RIBOSWITCH}

The S-box was one of the first mRNA regulatory elements described as a potential riboregulatory element. The sequence identified as the aptamer domain contains a number of phylogenetically conserved nucleotides that center within and surrounding a four-way junction motif (Fig. 6a). Biochemical and genetic analysis indicates that

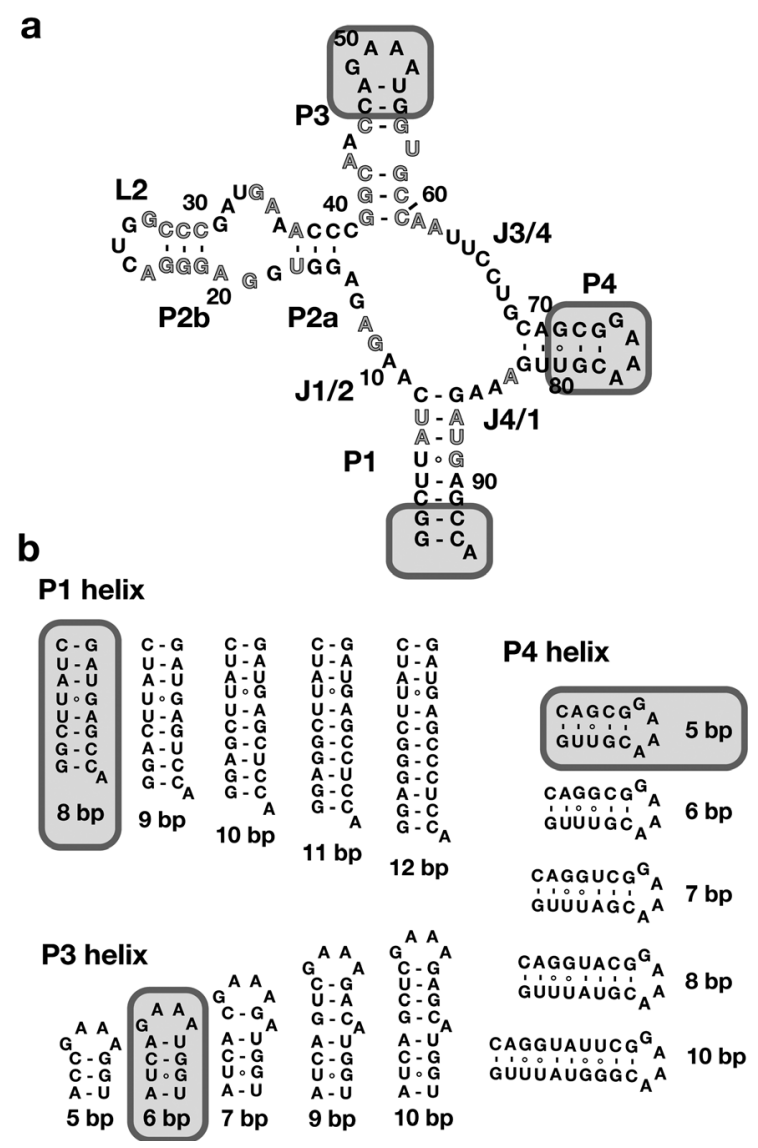

Figure 6. Secondary structure of the RNA ( $a$ ) used for the structure determination of the SAM-I riboswitch. Nucleotides that are $>95 \%$ conserved across phylogeny are depicted in gray outline. The boxed regions were the portions of sequence that are phylogenetically variable and thus systematically varied to create a library of RNAs tested for crystallizability. The library was created by varying the lengths of the P1, P3, and P4 helices (b), as well as changing the wild-type terminal loops of P3 and P4 to GAAA tetraloops. 
many of these conserved sequence elements are critical for the formation of a kink-turn motif and a pseudoknot that are crucial for metabolite binding and gene regulation, suggesting that this RNA adopts a complex tertiary architecture.

\section{Structure Determination}

To understand how this RNA specifically binds $S$ adenosylmethionine (SAM), we solved the liganded form of the S-box. The strategy that we used to find a crystallizable RNA was an extension of that used for the guanine riboswitch. From phylogenetic alignment of over 250 sequences from the Rfam database (Griffiths-Jones et al. 2003, 2005), it was clear that the conserved nucleotides were all contained in a defined four-way junction region (Fig. 6a). From a large number of sequences, we chose to focus on one from Thermoanaerobacter tengcongensis, a bacterium that grows optimally at approximately $70^{\circ} \mathrm{C}$, suggesting a highly stable RNA-ligand complex. Because thermophilic proteins are a popular target of crystallographers, presumably for their enhanced stability, we reasoned that their RNAs would share the same properties and thus tend to be more crystallizable. The termini of helices 1, 3, and 4 are all of variable length and sequence, indicating that they are nonessential for ligand binding and thus can be altered without affecting the RNA's SAM-binding activity. To find an appropriate RNA, we generated a library of individuals in which the length of these helices was systematically varied (Fig. 6b). Additionally, the sequence of the terminal loops of P3 and P4 were changed to GAAA, a tetraloop motif that has been shown to mediate crystal contacts in a number of RNA and RNP crystals (Pley et al. 1994; Batey et al. 2001). On the basis of these approaches, we created a library of approximately 70 variants by PCR and transcribed them into RNA using conventional methods.

Each variant was subjected to crystallization trials using four commercially available kits. These trials were allowed to incubate for 1 week at room temperature. Only constructs yielding single crystals that diffracted to $<5 \AA$ resolution in this initial screen were subjected to further trials; variants that failed this test were discarded. After investigation of a number of variants, it became clear that constructs in which the lengths of the P1, P3, and P4 helices were reduced to their minimal lengths yielded crystals in a significant number of conditions, indicating their increased propensity to crystallize as compared to RNAs with longer peripheral helices. Further screening around these initial hits using sequences with minor sequence changes yielded a construct that rapidly and reproducibly crystallized (Fig. 6b).

Again, the second consideration in our crystallization strategy was the phase problem. We used a strategy related to that used for the guanine riboswitch, relying on the ability of hexaammine compounds (cobalt, osmium, and iridium) to promiscuously, yet specifically, bind nucleic acids. In the case of the SAM riboswitch, once we found a construct that reproducibly yielded crystals, we reoptimized the crystallization conditions with iridium hexaammine. This yielded diffraction-quality crystals under conditions with $8 \mathrm{~mm}$ iridium hexaammine. To prepare the crystals for a MAD experiment, they were cryoprotected for 5 minutes in a solution containing $15 \%$ ethylene glycol, but no $\operatorname{Ir}\left(\mathrm{NH}_{3}\right)_{6} \mathrm{Cl}_{3}$, serving also as a brief back-soak to remove excess heavy atom. These crystals yielded a clearly interpretable Patterson map, and using CNS, we were able to rapidly find a four-heavy-atom solution that yielded sufficient phase information to calculate an interpretable electron density map.

\section{Structure of the SAM-I Riboswitch}

The S-box or SAM-I motif folds into a structure consisting of two sets of coaxially stacked helices (Montange and Batey 2006). Although it is tempting to call each of these "domains" in a fashion similar to those of the group I intron, we do not know whether each of these would be able to fold in the absence of the other or the stabilizing influence of single-stranded joining regions. The first coaxial stack is between $\mathrm{P} 1$ and $\mathrm{P} 4$ with the adenosine residues of the $\mathrm{J} 4 / 1$ joining region forming a contiguous helical stretch between the two A-form helices (Fig. 7). The second stack involves P2 and P3, which is broken by a canonical kink-turn motif (Fig. 7, KT) (Klein et al. 2001) that redirects the terminal loop L2 back toward the P1/P4 stack to form a pseudoknot with J3/4. Packing of the two sets of stacks is further facilitated by extensive tertiary interactions made with the $\mathrm{J} 1 / 2$ and $\mathrm{J} 3 / 4$ stands.

Using a bipartite site created by the minor grooves of the P1 and P3 helices, the RNA binds $S$-adenosylmethionine. The ligand itself is bound in a very compact configuration (the cis-configuration) in which the methionine moiety

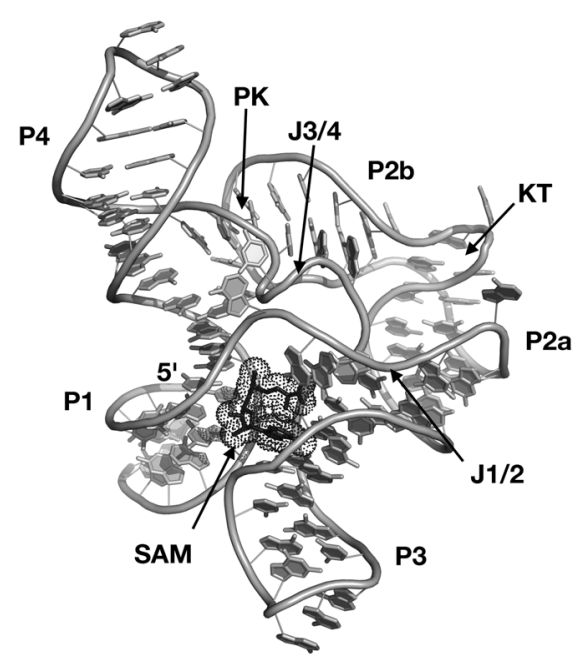

Figure 7. Global fold of the $S$-adenosylmethionine (SAM-I) riboswitch aptamer domain (PDB 2GIS). Labeling of the helices is the same as in Fig. 6a, along with the kink-turn (KT) and pseudoknot (PK) motifs. SAM is highlighted by a dotted surface representation (dark gray) which is found in a binding pocket formed by the minor grooves of P1 and P3 along with J1/2. 
a

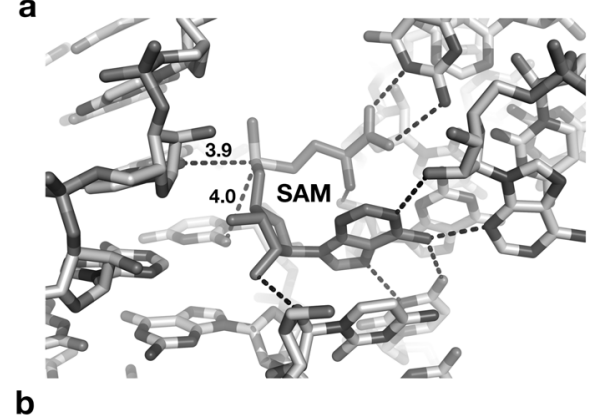

b.

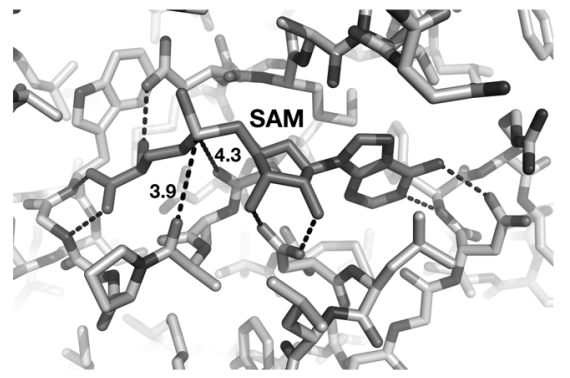

Figure 8. Comparison of the recognition of SAM by (a) RNA and by $(b)$ the methyltransferase FtsJ (PDB 1EJ0). All hydrogen bonds shown between SAM and the RNA or protein are standard distances $(2.8-3.3 \AA)$. The electrostatic interactions between the charged sulfur moiety and carbonyl atoms in the RNA or protein are also depicted by dashes with interatomic distances (in angstroms) between the sulfur atom and the carbonyl oxygen denoted.

stacks above the adenosyl purine ring (Fig. 8a). This stacking is presumably stabilized through a pi-cation interaction between the positively charged main-chain amino group of methionine and the aromatic ring system of the adenyl moiety. This is in contrast to most proteins that bind SAM in a trans-configuration (Fig. 8b), presumably to position the activated methyl group for transfer to a wide variety of substrates. The cis-configuration of SAM creates two different faces that interact with the P1 and $\mathrm{P} 3$ helices in very different ways. SAM is recognized by the minor groove of P3 via extensive hydrogen bonding with the adenine ring and the main-chain atoms of the methionine moiety. It is likely that these interactions serve as the bulk of the stabilizing interactions with the RNA.

The other face of SAM comprises the ribose sugar and the sulfur moiety. The presence of the activated methyl group is sensed through an electrostatic mechanism via the positive charge on the sulfur atom. Along with the ribose sugar, the sulfur packs against the $3^{\prime}$ side of the P1 helix, primarily interacting with two universally conserved A-U pairs. The positively charged sulfur group is placed approximately $4 \AA$ from two carbonyl groups $(\mathrm{O} 2)$ of the two uracils (Fig. 8a). This electrostatic interaction results in the S-box's approximately 100-fold preference for SAM over $S$-adenosylhomocysteine (SAH), which does not possess an activated methyl group. FtsJ, a methyltransferase, uses a similar recognition strategy for discrimination between the SAM cofactor and SAH (Fig. 8b) (Bugl et al. 2000).

A further consequence of SAM binding along the P1 helix is that it facilitates a series of tertiary interactions between the $3^{\prime}$ strands of the P1 and P3 helices. This small network of hydrogen bonds is clearly induced by the association of the RNA with SAM, as revealed by inline probing. Nucleotides on the $3^{\prime}$ side of the P1 helix show a significant degree of protection from magnesiuminduced backbone cleavage only in the presence of SAM, an indicator of ligand-induced RNA-RNA interactions. This ligand-induced tertiary structure is a critical component of SAM-dependent regulation, as these interactions serve to stabilize the $3^{\prime}$ side of the communication helix (P1) from being used to form an alternative secondary structure, the antiterminator helix. Thus, binding of SAM to the aptamer domain fates the expression platform to form a classic rho-independent terminator helix, causing transcription to stop.

\section{COMMON THEMES AMONG THE RIBOSWITCH STRUCTURES}

Currently, structures of purine (Batey et al. 2004; Serganov et al. 2004), $S$-adenosylmethionine (Montange and Batey 2006), and thiamine pyrophosphate (TPP) (Serganov et al. 2006; Thore et al. 2006) have been solved to atomic resolution. Each of these riboswitches is architecturally different, arising from their need to bind chemically distinct ligands. However, there are three critical similarities between the RNAs that we speculate are generalizable to all biological RNAs responsible for specifically binding small molecules.

Each of the riboswitch aptamer domains has tertiary architectural elements outside the ligand-binding pocket that serve to globally organize the RNA, in contrast to the majority of in-vitro-selected aptamers that bind small molecules. Typical in-vitro-selected aptamers, such as the ATP (Dieckmann et al. 1996; Jiang et al. 1996) and theophylline (Zimmermann et al. 1997) aptamers, bind their ligands through internal loop motifs embedded within single stem-loop structures. More complex folding involving helix-helix packing has not been structurally observed, although it likely occurs in the more complex in-vitro-selected ligase enzymes (Bartel and Szostak 1993; Bergman et al. 2004). Conversely, each riboswitch structure reveals at least one tertiary interaction via a loop-loop, pseudoknot, or loop-helix interaction in the purine, SAM, and TPP riboswitches, respectively. Disrupting mutations in these motifs result in loss of ligand binding in vitro, as well as the ability of the riboswitch to regulate gene expression in vivo. Because the global architecture of these riboswitch aptamer domains is established prior to ligand binding, this suggests that scaffolding of the binding pocket by tertiary architecture in a fashion akin to proteins is a common feature of these RNA elements. Recent studies of GTP aptamers by the Szostak group have suggested that increasing the informational complexity of the RNA is the route to greater specificity for a particular ligand (Carothers et al. 2006a, b), consistent with the observation of structurally complex biological aptamers.

A second commonality between these RNAs is the degree to which they recognize their cognate ligand. Unlike their in-vitro-selected kin, these RNAs appear to 
recognize the majority, if not all, of the functional groups in the ligand through direct and indirect readout mechanisms. This is exemplified by the SAM riboswitch, which recognizes its ligand through a series of hydrogen-bonding and base-stacking interactions with P3, Van der Waals, and electrostatic interactions with $\mathrm{P} 1$, and indirect readout to detect the presence of the methyl group and the number of methylene groups in the methionine side chain (Fig. 8a). Often the degree of recognition of ligands by in-vitro-selected aptamers is limited by the fact that their ligand was coupled to a column to effect a separation of binding and nonbinding RNAs.

Finally, and most importantly, is the coupling of ligand binding and tertiary structural changes in the aptamer domain. In each case, architectural changes in the RNA occur upon ligand binding that serves to stabilize a "communication helix," usually the P1 helix, that relays the liganded state of the aptamer domain to the expression platform. In the case of the $x p t-p b u X$ guanine riboswitch, binding of the ligand to the three-way junction induces a series of two base triples between nucleotides in $\mathrm{J} 2 / 3$ and base pairs in $\mathrm{P} 1$. This prevents the $3^{\prime}$ side of the $\mathrm{P} 1$ helix from being used to form the antiterminator element (which would allow complete synthesis of the mRNA transcript) and instead fates the mRNA to form a rhoindependent terminator element in the expression platform, aborting mRNA synthesis and shutting off gene expression (Fig. 1).

\section{CONCLUDING REMARKS}

Riboregulation by small noncoding RNAs and elements in the $5^{\prime}$ - and $3^{\prime}$-UTRs of mRNAs is now understood to be a major mechanism by which gene expression is controlled. In bacteria, riboswitches control a significant number of crucial metabolic pathways at the transcriptional and translational levels via their ability to bind a variety of metabolites. In the last few years, a significant amount of effort by our group and other workers has been devoted toward uncovering the molecular basis for this form of genetic control. Despite the determination of the structures of three of the aptamer domains bound to their cognate ligand, a number of fundamental questions remain as to how productive binding is achieved and its relationship to the expression platform.

Like many forms of RNA recognition, riboswitches depend on an "induced fit" mechanism for ligand binding in which the RNA undergoes a concerted binding and local folding event (Williamson 2000; Leulliot and Varani 2001). In the case of the purine riboswitch, we have presented a model of the process by which this may occur (Gilbert et al. 2006), but this mechanism still requires further experimental evidence to fully support it. The SAM and TPP riboswitches, instead of using a single binding pocket, have a "bipartite" pocket created by the union of two helices (P1 and P3 in the case of the SAM riboswitch) (Montange and Batey 2006; Serganov et al. 2006; Thore et al. 2006). In these cases, the ligand initially docks with one of these helices, and then the second closes around the initial ligand-RNA complex to fully encapsulate the ligand. In the case of the SAM riboswitch, it is likely that hydrogen bonding to the P3 helix precedes interactions with the P1 helix; in the case of the TPP riboswitch, the P2-J2/3-P3 coaxial stack which recognizes the pyrimidine moiety that likely initially interacts with the ligand. Detailed mechanistic analysis of these events may yield new insights into specific recognition of RNAs whose binding sites are disorganized in their free state.

Despite being locally disorganized in the free state, the binding pocket must have some structural cues that serve to guide productive ligand binding. In the case of the purine riboswitch, we have hypothesized that whereas most of the three-way junction is conformationally flexible, a critical pyrimidine residue (C/U74) is properly positioned for recognition by the purine nucleobase to initiate a productive binding process (Gilbert et al. 2006). Furthermore, we have accrued clear evidence that a number of nucleotides in and around the three-way junction have been evolutionarily selected, not because of their critical importance for the bound state, but rather to keep the free state open and receptive to ligand binding (C. Love et al., in prep.). This suggests that the structure of the unbound state is crucial for proper riboswitch function; that is, if the regulatory RNA has a tendency to misfold, the gene it controls may become constitutively activated or repressed. Thus, these RNAs are fine-tuned to avoid the "alternative conformational hell" first described by the Uhlenbeck lab that plagues a large number of RNAs in the laboratory (Uhlenbeck 1995). A comprehensive understanding of the free state of these RNAs will likely not come from NMR or X-ray crystallography, but rather from a combination of biophysical and biochemical methods that can more readily cope with dynamic states of the molecule.

A central question is, Exactly how does the RNA regulate gene expression through the interplay of the aptamer and expression platforms in the biological context? Biophysical studies by several groups have suggested that ligand binding and secondary structural rearrangements of the mRNA are slow processes (Wickiser et al. 2005a,b; Gilbert et al. 2006). If transcription by RNA polymerase proceeds through the 5'UTR at standard rate ( $\sim 60-90$ nucleotides/sec), then crucial steps for riboswitch function appear to be too slow for it to be an effective means of genetic control. It has been found that multiple transcriptional pause sites strategically placed within the FMN riboswitch significantly slow the rate of transcription through the regulatory element in vitro, but whether these pause sites play the same role in transcription regulation in vivo and are general to all riboswitches is unknown (Wickiser et al. 2005b).

This emphasizes the need for further structural and biophysical studies to specifically address the relationship between the aptamer domain and the expression platform. Because these domains are not tightly coupled through tertiary contacts, this will not come in the form of NMR or crystallographic structures, but rather through other biophysical techniques such as measuring force/extension curves using optical tweezers and singlemolecule FRET (Onoa and Tinoco 2004). These techniques have been particularly successful at understanding RNA folding/unfolding processes, and the riboswitch is 
inherently an RNA folding problem. Further complicating a biophysical characterization of this process, however, is that this ligand-influenced folding event is occurring co-transcriptionally such that decisions are likely being made prior to the entire 5'UTR being synthesized. Thus, it may be useful to study subfragments of the riboswitch that represent transcriptional intermediates, such as the aptamer/antiterminator region without an intact terminator sequence. Coupled with genetic and in vivo experimental results, answers to these questions will yield a clear picture as to how biology has harnessed mRNA to be able to both carry and regulate genetic information.

\section{ACKNOWLEDGMENTS}

The authors thank the members of the Batey laboratory for support in various aspects of our work on riboswitches, in particular Sarah Mediatore, Elizabeth Pleshe, Crystal Love, and Andrea Edwards. We also are grateful for engaging discussions and feedback from the Boulder RNA community and Professor Jeffrey Kieft. This work was supported by grants from the American Cancer Society and the National Institutes of Health.

\section{REFERENCES}

Adams P.L., Stahley M.R., Kosek A.B., Wang J., and Strobel S.A. 2004. Crystal structure of a self-splicing group I intron with both exons. Nature 430: 45.

Babitzke P. 2004. Regulation of transcription attenuation and translation initiation by allosteric control of an RNA-binding protein: The Bacillus subtilis TRAP protein. Curr. Opin. Microbiol. 7: 132.

Barrick J.E., Corbino K.A., Winkler W.C., Nahvi A., Mandal M., Collins J., Lee M., Roth A., Sudarsan N., Jona I., et al. 2004. New RNA motifs suggest an expanded scope for riboswitches in bacterial genetic control. Proc. Natl. Acad. Sci. 101: 6421.

Bartel D.P. and Szostak J.W. 1993. Isolation of new ribozymes from a large pool of random sequences (comment). Science 261: 1411 .

Batey R.T., Gilbert S.D., and Montange R.K. 2004. Structure of a natural guanine-responsive riboswitch complexed with the metabolite hypoxanthine. Nature 432: 411.

Batey R.T., Sagar M.B., and Doudna J.A. 2001. Structural and energetic analysis of RNA recognition by a universally conserved protein from the signal recognition particle. J. Mol. Biol. 307: 229.

Bergman N.H., Lau N.C., Lehnert V., Westhof E., and Bartel D.P. 2004. The three-dimensional architecture of the class I ligase ribozyme. $R N A$ 10: 176

Brunger A.T., Adams P.D., Clore G.M., DeLano W.L., Gros P., Grosse-Kunstleve R.W., Jiang J.S., Kuszewski J., Nilges M., Pannu N.S., et al. 1998. Crystallography \& NMR system: A new software suite for macromolecular structure determination. Acta Crystallogr. D Biol. Crystallogr. 54: 905.

Bugl H., Fauman E.B., Staker B.L., Zheng F., Kushner S.R., Saper M.A., Bardwell J.C., and Jakob U. 2000. RNA methylation under heat shock control. Mol. Cell 6: 349

Carothers J.M., Oestreich S.C., and Szostak J.W. 2006a. Aptamers selected for higher-affinity binding are not more specific for the target ligand. J. Am. Chem. Soc. 128: 7929.

Carothers J.M., Davis J.H., Chou J.J., and Szostak J.W. 2006b. Solution structure of an informationally complex high-affinity RNA aptamer to GTP. RNA 12: 567.

Carrasco N., Buzin Y., Tyson E., Halpert E., and Huang Z. 2004. Selenium derivatization and crystallization of DNA and RNA oligonucleotides for X-ray crystallography using multiple anomalous dispersion. Nucleic Acids Res. 32: 1638.
Corbino K.A., Barrick J.E., Lim J., Welz R., Tucker B.J., Puskarz I., Mandal M., Rudnick N.D., and Breaker R.R. 2005. Evidence for a second class of S-adenosylmethionine riboswitches and other regulatory RNA motifs in alphaproteobacteria. Genome Biol. 6: R70.

Cromie M.J., Shi Y., Latifi T., and Groisman E.A. 2006. An RNA sensor for intracellular $\mathrm{Mg}(2+)$. Cell 125: 71.

Dauter Z., Dauter M., and Dodson E. 2002. Jolly SAD. Acta Crystallogr. D Biol. Crystallogr. 58: 494.

Dieckmann T., Suzuki E., Nakamura G.K., and Feigon J. 1996. Solution structure of an ATP-binding RNA aptamer reveals a novel fold. RNA 2: 628 .

Ferre-D'Amare A.R. and Doudna J.A. 2000. Crystallization and structure determination of a hepatitis delta virus ribozyme: Use of the RNA-binding protein U1A as a crystallization module. J. Mol. Biol. 295: 541.

Ferre-D'Amare A.R., Zhou K., and Doudna J.A. 1998. Crystal structure of a hepatitis delta virus ribozyme. Nature 395: 567.

Gelfand M.S., Mironov A.A., Jomantas J., Kozlov Y.I., and Perumov D.A. 1999. A conserved RNA structure element involved in the regulation of bacterial riboflavin synthesis genes. Trends Genet. 15: 439.

Gilbert S.D., Stoddard C.D., Wise S.J., and Batey R.T. 2006. Thermodynamic and kinetic characterization of ligand binding to the purine riboswitch aptamer domain. J. Mol. Biol. 359: 754 .

Griffiths-Jones S., Bateman A., Marshall M., Khanna A., and Eddy S.R. 2003. Rfam: An RNA family database. Nucleic Acids Res. 31: 439.

Griffiths-Jones S., Moxon S., Marshall M., Khanna A., Eddy S.R., and Bateman A. 2005. Rfam: Annotating non-coding RNAs in complete genomes. Nucleic Acids Res. 33: D121.

Grundy F.J. and Henkin T.M. 1998. The S-box regulon: A new global transcription termination control system for methionine and cysteine biosynthesis genes in gram-positive bacteria. Mol. Microbiol. 30: 737.

Hendrickson W.A. and Ogata C.M. 1997. Phase determination from multiwavelength anomalous diffraction measurements. Methods Enzymol. 276: 494.

Hobartner C., Rieder R., Kreutz C., Puffer B., Lang K., Polonskaia A., Serganov A., and Micura R. 2005. Syntheses of RNAs with up to 100 nucleotides containing site-specific 2'methylseleno labels for use in X-ray crystallography. J. Am. Chem. Soc. 127: 12035.

Jiang F., Kumar R.A., Jones R.A., and Patel D.J. 1996. Structural basis of RNA folding and recognition in an AMP-RNA aptamer complex. Nature 382: 183.

Ke A. and Doudna J.A. 2004. Crystallization of RNA and RNAprotein complexes. Methods 34: 408.

Kieft J.S. and Batey R.T. 2004. A general method for rapid and nondenaturing purification of RNAs. RNA 10: 988.

Kieft J.S., Zhou K., Grech A., Jubin R., and Doudna J.A. 2002. Crystal structure of an RNA tertiary domain essential to HCV IRES-mediated translation initiation. Nat. Struct. Biol. 9: 370 .

Klein D.J., Schmeing T.M., Moore P.B., and Steitz T.A. 2001. The kink-turn: A new RNA secondary structure motif. EMBO J. 20: 4214.

Leulliot N. and Varani G. 2001. Current topics in RNA-protein recognition: Control of specificity and biological function through induced fit and conformational capture. Biochemistry 40: 7947.

Mandal M. and Breaker R.R. 2004a. Adenine riboswitches and gene activation by disruption of a transcription terminator. Nat. Struct. Mol. Biol. 11: 29.

. 2004b. Gene regulation by riboswitches. Nat. Rev. Mol. Cell Biol. 5: 451.

Mandal M., Boese B., Barrick J.E., Winkler W.C., and Breaker R.R. 2003. Riboswitches control fundamental biochemical pathways in Bacillus subtilis and other bacteria. Cell 113: 577.

Mandal M., Lee M., Barrick J.E., Weinberg Z., Emilsson G.M., Ruzzo W.L., and Breaker R.R. 2004. A glycine-dependent riboswitch that uses cooperative binding to control gene expression. Science 306: 275. 
McDaniel B.A., Grundy F.J., Artsimovitch I., and Henkin T.M. 2003. Transcription termination control of the $\mathrm{S}$ box system: Direct measurement of S-adenosylmethionine by the leader RNA. Proc. Natl. Acad. Sci. 100: 3083.

Miranda-Rios J., Navarro M., and Soberon M. 2001. A conserved RNA structure (thi box) is involved in regulation of thiamin biosynthetic gene expression in bacteria. Proc. Natl. Acad. Sci. 98: 9736.

Mironov A.S., Gusarov I., Rafikov R., Lopez L.E., Shatalin K., Kreneva R.A., Perumov D.A., and Nudler E. 2002. Sensing small molecules by nascent RNA: A mechanism to control transcription in bacteria. Cell 111: 747 .

Montange R.K. and Batey R.T. 2006. Structure of the $\mathrm{S}$-adenosylmethionine riboswitch regulatory mRNA element. Nature 441: 1172.

Nahvi A., Sudarsan N., Ebert M.S., Zou X., Brown K.L., and Breaker R.R. 2002. Genetic control by a metabolite binding mRNA. Chem. Biol. 9: 1043.

Noeske J., Richter C., Grundl M.A., Nasiri H.R., Schwalbe H., and Wohnert J. 2005. An intermolecular base triple as the basis of ligand specificity and affinity in the guanine- and adenine-sensing riboswitch RNAs. Proc. Natl. Acad. Sci. 102: 1372 .

Onoa B. and Tinoco I., Jr. 2004. RNA folding and unfolding. Curr. Opin. Struct. Biol. 14: 374.

Pflugrath J.W. 1999. The finer things in X-ray diffraction data collection. Acta Crystallogr. D Biol. Crystallogr. 55: 1718.

Pley H.W., Flaherty K.M., and McKay D.B. 1994. Model for an RNA tertiary interaction from the structure of an intermolecular complex between a GAAA tetraloop and an RNA helix. Nature 372: 111 .

Rice L.M., Earnest T.N., and Brunger A.T. 2000. Single-wavelength anomalous diffraction phasing revisited. Acta Crystallogr. D Biol. Crystallogr. 56: 1413.

Rodionov D.A., Vitreschak A.G., Mironov A.A., and Gelfand M.S. 2002. Comparative genomics of thiamin biosynthesis in procaryotes. New genes and regulatory mechanisms. J. Biol. Chem. 277: 48949.

- 2003. Regulation of lysine biosynthesis and transport genes in bacteria: Yet another RNA riboswitch? Nucleic Acids Res. 31: 6748 .

Rupert P.B. and Ferre-D'Amare A.R. 2001. Crystal structure of a hairpin ribozyme-inhibitor complex with implications for catalysis. Nature 410: 780.

Serganov A., Polonskaia A., Phan A.T., Breaker R.R., and Patel D.J. 2006. Structural basis for gene regulation by a thiamine pyrophosphate-sensing riboswitch. Nature 441: 1167.

Serganov A., Yuan Y.R., Pikovskaya O., Polonskaia A., Malinina L., Phan A.T., Hobartner C., Micura R., Breaker R.R., and Patel D.J. 2004. Structural basis for discriminative regulation of gene expression by adenine- and guaninesensing mRNAs. Chem. Biol. 11: 1729.

Stura E.A. and Wilson I.A. 1990. Analytical and production seeding techniques. Methods 1: 38 .
Sudarsan N., Barrick J.E., and Breaker R.R. 2003a. Metabolitebinding RNA domains are present in the genes of eukaryotes. RNA 9: 644.

Sudarsan N., Wickiser J.K., Nakamura S., Ebert M.S., and Breaker R.R. 2003b. An mRNA structure in bacteria that controls gene expression by binding lysine. Genes Dev. 17: 2688.

Teplova M., Wilds C.J., Wawrzak Z., Tereshko V., Du Q., Carrasco N., Huang Z., and Egli M. 2002. Covalent incorporation of selenium into oligonucleotides for X-ray crystal structure determination via MAD: Proof of principle. Multiwavelength anomalous dispersion. Biochimie 84: 849.

Terwilliger T.C. 2003. SOLVE and RESOLVE: Automated structure solution and density modification. Methods Enzymol. 374: 22.

Thore S., Leibundgut M., and Ban N. 2006. Structure of the eukaryotic thiamine pyrophosphate riboswitch with its regulatory ligand. Science 312: 1208.

Uhlenbeck O.C. 1995. Keeping RNA happy. RNA 1: 4.

Vitreschak A.G., Rodionov D.A., Mironov A.A., and Gelfand M.S. 2002. Regulation of riboflavin biosynthesis and transport genes in bacteria by transcriptional and translational attenuation. Nucleic Acids Res. 30: 3141.

Wickiser J.K., Cheah M.T., Breaker R.R., and Crothers D.M. 2005a. The kinetics of ligand binding by an adenine-sensing riboswitch. Biochemistry 44: 13404.

Wickiser J.K., Winkler W.C., Breaker R.R., and Crothers D.M. $2005 \mathrm{~b}$. The speed of RNA transcription and metabolite binding kinetics operate an FMN riboswitch. Mol. Cell 18: 49.

Williamson J.R. 2000. Induced fit in RNA-protein recognition. Nat. Struct. Biol. 7: 834.

Winkler W.C. and Breaker R.R. 2005. Regulation of bacterial gene expression by riboswitches. Annu. Rev. Microbiol. 59: 487.

Winkler W.C., Cohen-Chalamish S., and Breaker R.R. 2002a. An mRNA structure that controls gene expression by binding FMN. Proc. Natl. Acad. Sci. 99: 15908.

Winkler W.C., Nahvi A., and Breaker R.R. 2002b. Thiamine derivatives bind messenger RNAs directly to regulate bacterial gene expression. Nature 419: 952.

Winkler W.C., Nahvi A., Roth A., Collins J.A., and Breaker R.R. 2004. Control of gene expression by a natural metaboliteresponsive ribozyme. Nature 428: 281.

Winkler W.C., Nahvi A., Sudarsan N., Barrick J.E., and Breaker R.R. 2003. An mRNA structure that controls gene expression by binding S-adenosylmethionine. Nat. Struct. Biol. 10: 701.

Zengel J.M. and Lindahl L. 1994. Diverse mechanisms for regulating ribosomal protein synthesis in Escherichia coli. Prog. Nucleic Acid Res. Mol. Biol. 47: 331.

Zhang L. and Doudna J.A. 2002. Structural insights into group II intron catalysis and branch-site selection. Science 295: 2084.

Zimmermann G.R., Jenison R.D., Wick C.L., Simorre J.P., and Pardi A. 1997. Interlocking structural motifs mediate molecular discrimination by a theophylline-binding RNA. Nat. Struct. Biol. 4: 644. 


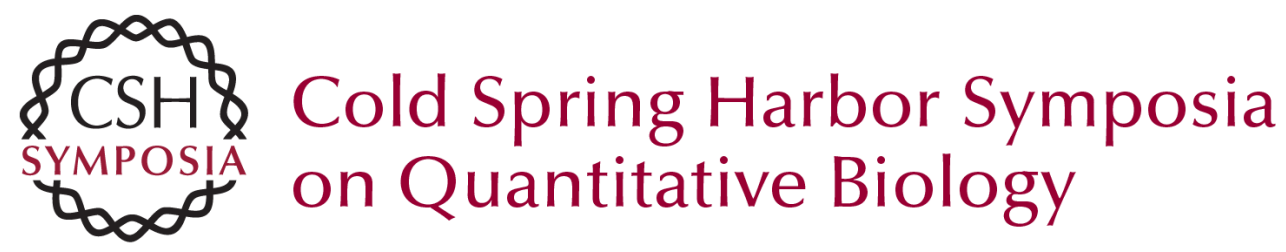

\section{Structural Studies of the Purine and SAM Binding Riboswitches}

S.D. GILBERT, R.K. MONTANGE, C.D. STODDARD, et al.

Cold Spring Harb Symp Quant Biol 2006 71: 259-268

Access the most recent version at doi:10.1101/sqb.2006.71.015

References This article cites 69 articles, 16 of which can be accessed free at: http://symposium.cshlp.org/content/71/259.full.html\#ref-list-1

License

Email Alerting Receive free email alerts when new articles cite this article - sign up in Service the box at the top right corner of the article or click here. 